It will be noticed that the value of the period for $19 \mathrm{I}_{3}$ depends on an interval of less than one year. A progressive lengthening of the period is perhaps shown by the above table.

The recorded times, throughout, are $6^{\mathrm{h}} \mathrm{o}^{\mathrm{m}}$ slow of Gr. M. T.

Yerkes Observatory, Williams Bay, Wisconsin, I 9 I 3 July 14.

E. E. Barnard.

\title{
Zur Frage über die Anzahl der Lösungen bei der Bestimmung einer parabolischen Bahn.
}

In meinem Aufsatze (A. N. 4495) habe ich gezeigt, daß, wenn bei der Bestimmung einer parabolischen Bahn auch die Bedingung der Ebene befriedigt wird, nicht mehr als zwei Lösungen existieren können. Jetzt will ich beweisen, daß von den beiden Lösungen, die man für die Distanz bekommt, nur eine Lösung positiv, die andere aber immer negativ ist.

Aus dem erwähnten Aufsatze nehme ich die Gl. (15) in der Form $\left[\left(\varrho-a_{1}\right)^{2}+a_{2}{ }^{2}\right]^{3}=b_{1} \varrho+b_{2}$.

Nach (12) und (14) ist

$$
\begin{array}{ll}
a_{1}=(g / h) \cos \varphi & a_{2}=(g / h) \sin \varphi \\
b_{0}=2^{2} \theta_{1}^{2} h^{-2} & b_{2}=D \cdot b_{0}^{3} \quad D=1 /\left(R_{2}+R\right)^{3} .
\end{array}
$$

Das von $\varrho$ unabhängige Glied der Gleichung, das ich mit $E$ bezeichnen werde, wird also sein

$$
E=\left(a_{1}{ }^{2}+a_{2}^{2}\right)^{3}-b_{2}
$$

oder, wenn man $a_{1}, a_{2}$ und $b_{2}$ einsetzt,

$$
E=(g / h)^{6}-2^{6} \theta_{1}{ }^{6} h^{-6} /\left(R_{2}+R\right)^{3}
$$

und dieser Ausdruck ist das Produkt aller sechs Wurzeln. Unter diesen sind vier Wurzeln imaginär und haben keinen Einfluß auf das Zeichen des Produktes (weil sie paarweise konjugiert sind). Das Zeichen hängt also ausschließlich von den beiden reellen Wurzeln ab. Ist dieses Produkt negativ, Kiew, Sternwarte, I9I3 Mai. so existiert nur eine positive Lösung, die andere ist also negativ.

Die Größe $g$ ist die Chorde der Erdbahn (genauer die Distanz $z$ wischen dem ersten und dem dritten locus fictus).

Wenn man die Exzentrizität der Erdbahn vernachlässigt und die Kleinheit von $g$ berücksichtigt, so kann man $g$ für einen Kreisbogen halten, der in $t_{2}-t$ Tagen von der Erde beschrieben ist. Nimmt man für den Radius dieser Kreisbahn die Größe $R_{1}=1 / 2\left(R_{2}+R\right)$, so hat man

$$
g=\frac{k\left(t_{2}-t\right)}{R_{1}^{3 / 2}} \cdot R_{1}=\frac{\theta_{1}}{V R_{1}}=\frac{\theta_{1} V_{2}}{V\left(R_{2}+R\right)} .
$$

Dieser Ausdruck, in $E$ eingesetzt, ergibt

$E=\frac{2^{3} \theta_{1}^{6}}{\left(R_{2}+R\right)^{3} h^{6}}-\frac{2^{6} \theta_{1}{ }^{6}}{\left(R_{2}+R\right)^{3} h^{6}}=-5^{6} \theta_{1}{ }^{6}\left(R_{2}+R\right)^{-3} h^{-6}$ oder, wenn $\operatorname{man} R_{2}+R=2$ setzt

$$
E=-7 \theta_{1}^{6} h^{-6} \text {. }
$$

Es ist also $E$ immer negativ, und demnach ist eine von den reellen Wurzeln der Gleichung (15) positiv, die andere immer negativ.

Daraus folgt, daß bei einer theoretisch vollständigen

\begin{tabular}{|c|c|c|c|c|}
\hline Planet & \multicolumn{2}{|c|}{1913} & \multicolumn{2}{|c|}{ M. Z. Gr } \\
\hline 72 Feronia & Aug. & 26 & & $22 \mathrm{~m}: 8$ \\
\hline 470 Kilia & 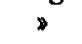 & 26 & & » \\
\hline I 35 Hertha & $>$ & 26 & & " \\
\hline 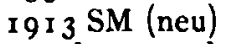 & » & 27 & 9 & 16.4 \\
\hline $713[1911 \mathrm{LS}]$ & 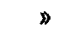 & 27 & & ” \\
\hline 96 Aegle & $»$ & 27 & & » \\
\hline 1 36 Austria & $»$ & 27 & & » \\
\hline I 68 Sibylla & 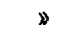 & 27 & & 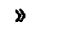 \\
\hline 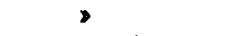 & 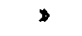 & 28 & 9 & $37 \cdot 5$ \\
\hline$I_{9}$ I $\left._{3} \mathrm{SL}(\text { neu })^{1}\right)$ & $>$ & 28 & & $»$ \\
\hline $732\left[\begin{array}{llll}19 & \text { I OR }\end{array}\right]$ & » & 28 & & 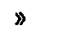 \\
\hline 313 Chaldaea & , & 28 & & • \\
\hline 50 Virginia ${ }^{2}$ ) & > & 28 & & $»$ \\
\hline 3 Juno & 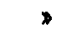 & 28 & & \# \\
\hline
\end{tabular}
Bestimmung einer parabolischen Bahn praktisch nur eine Lösung existiert.

$$
\text { R. Vogel. }
$$

\section{Mitteilungen utber Kleine Planeten.}

Photographische Aufnahmen in Simeis.

Simeìs, 19I3 Aug. 30.

2) Vergleiche die Auffindung von demselben Tage in Königstuhl A. N. 195.486. Rechen-Institut.

2) Eine Änderung von $M$ um -1 ㄴ genugt, um die starke $\mathrm{Ab}$ weichung des Jahrbuchorts zum Verschwinden zu bringen. Rech.-Inst.
Photographic Observations at Johannesburg. Planet I913 Gr.m.t. RA.1913.0 Decl. 1913.0 Magn. April

376 Geometria $27.33^{66}$ I $^{\mathrm{h}} 3^{2^{\mathrm{m}}} 3^{8^{\mathrm{s}} .25}-2 \mathrm{I}^{\circ} 28^{\prime} 23^{\prime \prime} \circ \mathrm{I} 0^{\mathrm{m}} \cdot 9$ May

$705[$ I910KV] $\quad 3.3078$ I $344 \quad 34.28-3528 \quad 58.8 \quad$ I 2.I 476 Hedwig $\quad 3.3078$ I4 $\quad$ I $13.72-2953 \quad 4.6$ I I.0 454 Mathesis $\quad 9.43$ I 7 I $434 \quad 59.10-$ I 740 I 3.4 II.5 Union Observatory, Johannesburg, I 9 I 3 August I I.

H. E. Wood.

Aufnahmen auf der Königstuhl-Sternwarte. I 9 I 3 Sept. 7 .

Planet Position I913.0 Tägl. Bew. Gr. Platte 624 Hektor $22^{\mathrm{h}} 34^{\mathrm{m}} \cdot 6-10^{\circ} 47^{\prime}-0^{\mathrm{m}} \cdot 5-2^{\prime} \times 3^{\mathrm{m}} \cdot 5$ D III5 I9I3 SK $22 \quad 32.9-1046-0.7-2 \quad 16 \quad$ * 248 Lameia $04.6+74-0.9-5$ I 3.I B 3275 659 Nestor nicht am Ephemeriden-Ort DII27
Platte m. Z. Kgst. Plattenmitte Beob.
B $3275 \quad I^{\mathrm{h}} 53^{\mathrm{m}} \cdot 7 \quad 23^{\mathrm{h}} 5^{8^{\mathrm{m}}}+6^{\circ} 20^{\prime} \quad F$. Kaiser
$\mathrm{DII25}$ I0 $59.52230 \quad-117 \%$ M. Wolf II27 I2 43.0 I 18 + IO 50 "

HeidelDerg, Königst.-Sternw., 19r 3 Sept. 8. M. Wolf. 